\title{
Mechanistic studies on the absorption enhancement of a self-nanoemulsifying drug delivery system loaded with norisoboldine-phospholipid complex
}

This article was published in the following Dove Press journal: International Journal of Nanomedicine

\author{
Jing Zhang ${ }^{1, *}$ \\ Xiaoxia Wen ${ }^{1} * *$ \\ Yue Dai ${ }^{2}$ \\ Yufeng Xia'
}

'Department of Pharmacognosy, School of Traditional Chinese Pharmacy, China Pharmaceutical University, Nanjing 2I I 198, People's Republic of China; ${ }^{2}$ Department of Traditional Chinese Medicine and Pharmacology, School of Traditional Chinese Pharmacy, China Pharmaceutical University, Nanjing 21II98, People's Republic of China

*These authors contributed equally to this work
Correspondence: Yufeng Xia

Department of Pharmacognosy, School of Traditional Chinese Pharmacy, China

Pharmaceutical University, 639 Longmian

Avenue, Jiangning District, Nanjing

211198, China

Tel +862583271400

Fax +86 2585301528

Email yfxiacpu@।26.com

Yue Dai

Department of Traditional Chinese Medicine and Pharmacology, School of Traditional Chinese Pharmacy, China

Pharmaceutical University, 639 Longmian

Avenue, Nanjing 21 II98, China

Email yuedaicpu@I26.com
Background: Norisoboldine (NOR), the main isoquinoline alkaloid constituent in Radix Linderae, was demonstrated to have an outstanding anti-arthritis activity. However, a poor oral bioavailability of NOR creates a barrier for its development and application.

Methods: A new self-nanoemulsifying drug delivery system (SNEDDS) loaded with the phospholipid complex (PC) was designed to improve the oral bioavailability of NOR. NOR-PC was prepared by solvent evaporation method with a mixture of phospholipid and NOR at a mass ratio of $3: 1$. The property of PC is to improve the liposolubility of NOR, and made PC embedded in the drug delivery system. The physicochemical property of NOR-PC was characterized by differential scanning calorimetry (DSC) and Fourier transform infrared spectroscopy (FT-IR). According to the ability to dissolve NOR-PC, the oil and cosurfactant were chosen. The surfactant was selected based on its emulsification efficiency in SNEDDS. Pseudo-ternary phase diagram was created to select the best formulation of NOR-PC-SNEDDS, and the pharmacokinetic parameters were detected in rats. In addition, intestinal lymphatic transport and liver microsome experiment were studied to gain insight into the mechanism for NOR-PC-SNEDDS increasing the oral bioavailability of NOR.

Results: Solubility detection showed that the PC significantly improved the liposolubility of NOR. NOR-PC-SNEDDS was prepared using NOR-PC, Ethyl oleate, Labrasol, Cremophor EL and transcutol HP at a weight ratio of 1:2:3.36:2.24:2.4 (w/w/w/w/w). The particle size and zeta potential of NOR-PC-SNEDDS were $36.72 \pm 1.47 \mathrm{~nm}$ and $-4.91 \pm 0.49 \mathrm{mV}$ after dilution with distilled water at a ratio of 1:50 (w/w). The absolute bioavailability of NOR in the NOR-PC-SNEDDS group significantly increased and the value was $372 \%$ in relative to NOR group. Further studies indicated that NOR-PC-SNEDDS promoted the oral bioavailability of NOR by enhancing intestinal lymphatic absorption and inhibiting Phase II metabolism of NOR.

Conclusion: These findings suggested that NOR-PC-SNEDDS was able to promote the oral bioavailability of NOR, which provided a foundation for the further development and application of NOR.

Keywords: norisoboldine, oral bioavailability, self-nanoemulsifying drug delivery system, lymphatic absorption, Phase II metabolism

\section{Introduction}

Rheumatoid arthritis (RA) is a common systemic autoimmune disease characterized by chronic synovial inflammation and hyperplasia as well as joint destruction and ultimate disability. Epidemiologic studies show that RA may increase the risk of 
comorbidities, such as cardiovascular disease, osteoporotic fracture and malignancy, and results in significant mortality. The prevalence of RA is estimated to be about $1 \%$ of the global population and $0.42 \%$ of the population in People's Republic of China. ${ }^{1,2}$ Currently, the disease-modifying anti-rheumatic drugs, non-steroidal anti-inflammatory drugs, and glucocorticoid are widely used for the treatment of RA. But their clinical applications were limited by the annoying side effects and the high costs. Therefore, there is an urgent need to find a novel candidate agent for the treatment of RA.

Norisoboldine (NOR, Figure 1) is an isoquinoline alkaloid isolated from the dry root of Lindera aggregate (Sims) Kosterm, which exhibited outstanding anti-arthritis activity. ${ }^{3-5}$ Previous studies in our laboratory showed that NOR (15 and $30 \mathrm{mg} / \mathrm{kg}$, oral) significantly decreased the swelling of paws and arthritis index scores, and rescued the body weight loss of mice with collagen-induced arthritis. ${ }^{4}$ The anti-arthritic mechanism of NOR involved the restoration of the balance between Th17 and regulatory T cells. ${ }^{6}$ Furthermore, synovial angiogenesis is considered as a potential target for the treatment of RA. Our findings demonstrated that NOR was able to prevent synovial angiogenesis in rats by moderating the Notch1 pathwayrelated endothelial tip cell phenotype with a potential action target of the Notch1 transcription complex. ${ }^{7,8}$ Therefore, NOR might become a promising candidate drug for the treatment of RA in view of the action mode of multiple targets and low toxic side effects. However, pharmacokinetic studies showed that the oral

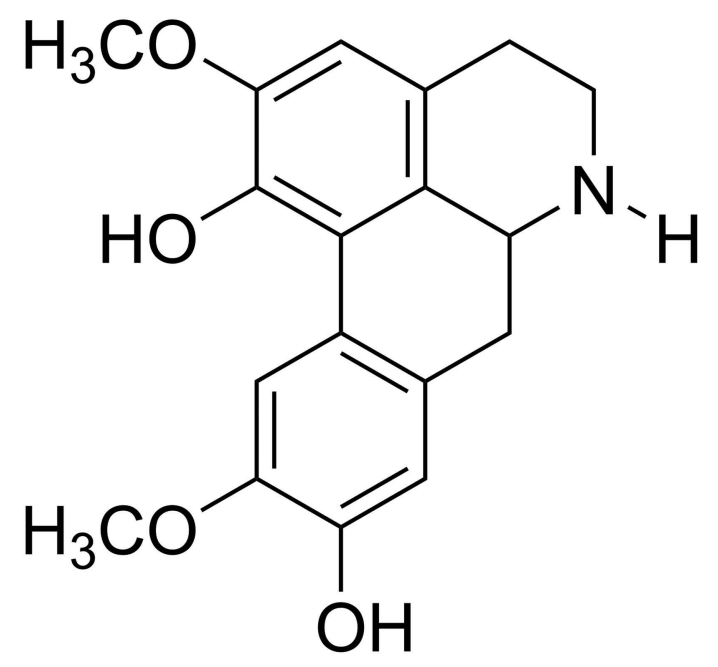

Figure I Chemical structure of NOR. Abbreviation: NOR, norisoboldine. bioavailability of NOR was only $2.77 \%,{ }^{9}$ which limit the further development of NOR.

Self-nanoemulsifying drug delivery system (SNEDDS) has been commonly used to improve the bioavailability of insoluble drugs. ${ }^{10-12}$ SNEDDS is a uniform and transparent solution composed of drug, oil, surfactant, and cosurfactant. SNEDDS can spontaneously form isotropic, transparent,or translucent solution in water with mild agitation at ambient temperature (usually at $37^{\circ} \mathrm{C}$ ). SNEDDS improves the oral bioavailability of the drugs by increasing solubility, accelerating intestinal permeability, facilitating intestinal lymphatic absorption, and attenuating gastrointestinal metabolism. ${ }^{13-15}$ Notably, NOR is unable to be embedded in SNEDDS without any treatment due to the low liposolubility. Previous reports indicated that phospholipid complex (PC) technique could improve the liposolubility of water-soluble drugs, and made them embedded in the drug delivery system. ${ }^{16-18}$ Therefore, NOR-PC was used to improve the liposolubility of NOR and prepared into NOR-PC-SNEDDS in combination with optimized excipients of SNEDDS.

To sum up, the aim of the present study was to design a new SNEDDS loaded with the PC to improve the oral bioavailability of NOR. Pharmacokinetic comparisons of the NOR, NOR-PC, and NOR-PC-SNEDDS were performed, and the oral bioavailability of NOR-PCSNEDDS was investigated. Furthermore, the mechanism for the absorption enhancement of NOR-PC-SNEDDS was explored.

\section{Materials and methods Materials}

NOR (purity $>98 \%$ ) was purchased from Chengdu Chroma Biotechnology Co., Ltd. (Chengdu, People's Republic of China). soybean phospholipid (95\% phosphatidylcholine) was donated by Shanghai Avet Pharmaceutical Technology Co., Ltd. (Shanghai, People's Republic of China). Labrafac Lipophile WL1349, Labrafil M 1944 CS, Peceol, Capryol 90, Lauroylycol 90, Labrasol, and Transcutol HP (analytical purity) were kindly supplied by Gattefossé (Shanghai, People's Republic of China). Cremophor EL, Cremophor RH 40, and Solutol HS 15 (analytical purity) were donated by BASF (Shanghai, People's Republic of China). Oleic acid and tween 80 (analytical purity) were purchased from Nanjing Chemical Reagent Co., Ltd. (Nanjing, People's Republic of China). Ethyl oleate and $\mathrm{MgCl}_{2}$ (analytical purity) were obtained from Sinopharm Chemical Reagent Co., Ltd. 
(Shanghai, People's Republic of China). UDPGA (purity $>93 \%$ ), D-Saccharic acid 1, 4-lactone (purity >98\%), and alamethicin (purity $>98 \%$ ) were purchased from SigmaAldrich (St. Louis, MO, USA). BCA Protein Assay Kit was purchased from Nanjing Jiancheng Bioengineering Insitute (Nanjing, People's Republic of China). Sinomenine (purity $>98 \%$, internal standard) was purchased from Nanjing Jingzhu Bio-technology Co., Ltd. HPLC-grade acetonitrile was purchased from Merck (Darmstadt, German). All other chemicals and solvents were of analytical purity.

\section{Animals}

Sprague-Dawley rats (180-220 g) were obtained from Nanjing Qinglongshan Animal Breeding Center (Nanjing, People's Republic of China). Rats were housed under controlled conditions of temperature $\left(22 \pm 2^{\circ} \mathrm{C}\right)$ and relative humidity $(50 \pm 10 \%)$ with a 12 -hr light/dark cycle. All rats had free access to food and sterile water for 7 days. All animal experiments were performed in accordance with the National Institutes of Health Guide for the Care and Use of Laboratory Animals, with the approval of the Animal Ethics Committee of School of Traditional Chinese Pharmacy, China Pharmaceutical University. Before experiments, rats were fasted for $12 \mathrm{hrs}$ while water was taken freely.

\section{Preparation of NOR-PC}

Soybean phospholipid and NOR at a mass ratio of 3:1 were mixed to prepare NOR-PC. Required amounts of NOR and phospholipids were placed in a $100 \mathrm{~mL}$ roundbottom flask and dissolved in absolute ethanol. The concentration of NOR was $1.25 \mathrm{mg} / \mathrm{mL}$. The mixture was then stirred with a magnetic mixer at $40^{\circ} \mathrm{C}$ for $3 \mathrm{hrs}$. Afterward, a vacuum rotary evaporator was used to evaporate solvent, and the residue was dried under vacuum for $24 \mathrm{hrs}$. The resultant NOR-PC was transferred into a glass bottle filled with nitrogen and stored at room temperature.

\section{Characterization of NOR-PC}

The physicochemical property of NOR-PC was characterized by differential scanning calorimetry (DSC) and Fourier transform infrared spectrophotometry (FT-IR). DSC analyses of NOR, phospholipid, NOR-PC, and physical mixture of NOR and phospholipid were recorded using a Netzsch DSC 204 F1 phoenix differential scanning calorimeter (Netzsch, Selb, Germany), respectively. The samples were heated from $40^{\circ} \mathrm{C}$ to $300^{\circ} \mathrm{C}$ at a heating rate of $10^{\circ} \mathrm{C} /$ min. The FT-IR spectra of the examined samples were conducted in the range of $4000-400 \mathrm{~cm}^{-1}$ with a resolution of $4 \mathrm{~cm}^{-1}$ using a Bruker tensor 27 FT-IR spectrometer (Bruker Corporation, Billerica, MA, USA). The examined samples were obtained employing compressed disks of appropriate amount of NOR, phospholipid, NOR-PC, and physical mixture of NOR and phospholipid separately mixed with $\mathrm{KBr}$ in the dry environment.

\section{Determination of NOR content in NOR-PC}

HPLC method was established to determine the concentration of NOR in NOR-PC. HPLC analysis was carried out on a Shimadzu LC-2010AHT HPLC system equipped with an ODS- $\mathrm{C}_{18}$ column $(150 \mathrm{~mm} \times 6 \mathrm{~mm}, 5 \mu \mathrm{m})$ (Shimpack Technologies, Kyoto, Japan). The mobile phase was acetonitrile: $0.1 \%$ formic acid water $(15: 85, \mathrm{v} / \mathrm{v})$ at a flow rate of $1 \mathrm{~mL} / \mathrm{min}$ and the injection volume was $20 \mu \mathrm{L}$. Column temperature and detection wavelength were set at $30^{\circ} \mathrm{C}$ and $280 \mathrm{~nm}$, respectively.

\section{Solubility studies}

The solubility of NOR-PC in double distilled water and n-octanol was measured to check whether the liposolubility of NOR was increased after the preparation of NOR-PC. Excess NOR, NOR-PC, and physical mixture of NOR and phospholipids were separately added to $5 \mathrm{~mL}$ double distilled water or n-octanol. The samples were shaken at $25 \pm 2^{\circ}$ C for $24 \mathrm{hrs}$ and then centrifuged at 12,000 rpm for 20 mins. Then, the supernatants were taken and diluted with methanol:0.1\% formic acid water $(3: 2, \mathrm{v} / \mathrm{v})$. The concentration of NOR was determined by the above HPLC method.

The solubility of NOR-PC in different oil phases, surfactants, and co-surfactants were determined to select proper formulation of NOR-PC-SNEDDS. One milliliter of various oil phases, surfactants, and co-surfactants were accurately measured and mixed with excess NOR-PC or NOR. The mixture was vortex-mixed for $1 \mathrm{~min}$ and shaken for $48 \mathrm{hrs}$ at $37^{\circ} \mathrm{C}$. The samples were centrifuged at 12,000 rpm for 20 mins. The supernatant $(10 \mu \mathrm{L})$ was collected and diluted with $990 \mu \mathrm{L}$ hydrochloric acid-methanol $(0.5 \%, \mathrm{v} / \mathrm{v})$. The mixture was vortex-mixed for $1 \mathrm{~min}$ and determined by HPLC method.

\section{Construction of pseudo-ternary phase diagram}

Ethyl oleate was selected as the oil phase, Labrasol: Cremophor EL (3:2) as the surfactant and Transcutol HP 
as the cosurfactant according to the results of the preliminary experiments. The amount of oil phase was fixed at $10-90 \%(w / w)$ and the ratio of surfactant to co-surfactant was from 1:9 to 9:1 to screen prescriptions. The mixture was stirred with a magnetic agitator for 20 mins. NOR-PC was added to the drug-loaded prescriptions before mixture.

Twenty-five milliliters of $0.1 \mathrm{M} \mathrm{HCl}$ solution was stirred with a magnetic agitator at $37^{\circ} \mathrm{C}$ for a low speed. A quantity of $100 \mu \mathrm{L}$ of the above-mixed solution was added to form a self-emulsified area with transparent or microstrip blue opalescence. Analysis software Origin 8.0 was used to construct the pseudo-ternary phase diagrams.

\section{Characterization of NOR-PC-SNEDDS}

The particle size, percent polydispersity, and zeta potential of the optimized NOR-PC-SNEDDS after dilution with distilled water $(1: 50, \mathrm{v} / \mathrm{v})$ were measured by a Malvern Zetasizer Nano ZS90 (Malvern Instruments, UK). Each sample was assayed for three times.

The morphological features of the NOR-PC-SNEDDS were observed by a Hitachi HT7700 transmission electron microscope (Hitachi Ltd.). The NOR-PC-SNEDDS was diluted with distilled water $(1: 50, \mathrm{v} / \mathrm{v})$ and mixed with vortex. One drop of the sample was placed on the copper sheet and negatively stained by $2 \%$ phosphomolybdic acid for $30 \mathrm{~s}$ prior to observation.

\section{Thermodynamic stability of NOR-PC-SNEDDS}

The effects of different media and dilution factors on the particle size and polydispersity index (PDI) of NOR-PCSNEDDS were investigated, respectively. NOR-PCSNEDDS was diluted with distilled water, $\mathrm{pH} 1.2 \mathrm{HCl}$ solution $(0.1 \mathrm{M})$, and $\mathrm{pH} 6.8$ phosphate buffer to 50 , 100 , and 1000 folds at $37^{\circ} \mathrm{C}$, respectively. ${ }^{16,19}$ Then, the particle size and PDI were determined using a Malvern Zetasizer Nano ZS90 to investigate the stability.

The effects of freeze-thaw on particle size and PDI were further investigated. After NOR-PC-SNEDDS was chilled for $24 \mathrm{hrs}$ at $-20^{\circ} \mathrm{C}$, it was placed at room temperature for $24 \mathrm{hrs}$, then freezed and thawed repeatedly for three times. Next, it was diluted 250 folds with distilled water. The particle size and PDI were measured to examine the thermodynamic stability.

\section{Pharmacokinetic studies of NOR in rats}

Rats were randomly divided into four groups (five rats in each group), including NOR group, NOR-PC group, NOR-PC-SNEDDS group, and NOR-IV group. The first three groups were administered by gavage at a dose equivalent to $30 \mathrm{mg} / \mathrm{kg}$ of NOR. Blood samples $(300 \mu \mathrm{L})$ were collected into heparinized tubes via the oculi choroidea vein at $0,0.083,0.17,0.33,0.5,0.75,1,1.5,2,3,4$, 8,12 , and $24 \mathrm{hrs}$ after oral administration. NOR-IV group was administered by tail vein injection at a dose equivalent to $2 \mathrm{mg} / \mathrm{kg}$ of NOR. Blood samples $(300 \mu \mathrm{L})$ were collected from the oculi choroidea veins at $0,0.033,0.083$, $0.17,0.33,0.5,0.75,1,1.5,2,3,4,8,12$, and $24 \mathrm{hrs}$. The obtained blood samples were centrifuged immediately at $8000 \mathrm{rpm}$ for $10 \mathrm{mins}$ at $4^{\circ} \mathrm{C}$, and stored at $-20^{\circ} \mathrm{C}$ until analysis.

The plasma samples were thawed at room temperature prior to processing. A $100 \mu \mathrm{L}$ of plasma sample was transferred to an Eppendorf tube, and $40 \mu \mathrm{L}$ of IS (300 ng/mL) was spiked and mixed for $30 \mathrm{~s}$. Then, $460 \mu \mathrm{L}$ methanol was added, and the mixture was thoroughly vortexed. After centrifugation at $12,000 \mathrm{rpm}$ for 10 mins, the supernatant $(400 \mu \mathrm{L})$ was transferred to a centrifuge tube and evaporated to dryness in a vacuum concentrator at $37^{\circ} \mathrm{C}$. The obtained residue was reconstituted in $50 \mu \mathrm{L} 0.1 \%$ formic acid water and centrifuged at $12,000 \mathrm{rpm}$ for $10 \mathrm{mins}$. The supernatant was used for LC-MS/MS analysis.

The samples were analyzed using an Agilent 1260 liquid chromatography (Agilent Technologies, Santa Clara, CA, USA) with an Agilent 6420 triple quadrupole mass spectrometer (Agilent Technologies). Chromatographic separation was performed on a ZORBAX Extend- $\mathrm{C}_{18}$ column $(2.1 \times 50$ $\mathrm{mm}, 1.8 \mu \mathrm{m}$ ) (Agilent Technologies). The mobile phase consisting of acetonitrile: $0.1 \%$ formic acid water $(9: 91, \mathrm{v} /$ v) was delivered at a flow rate of $0.2 \mathrm{~mL} / \mathrm{min}$. The injection volume was $5 \mu \mathrm{L}$. The electrospray ionization-MS/MS operation parameters were set as follows: positive ion electrospray; drying gas $\left(\mathrm{N}_{2}\right)$ temperature, $300^{\circ} \mathrm{C}$; drying gas $\left(\mathrm{N}_{2}\right)$ flow rate, $11 \mathrm{~L} / \mathrm{min}$; capillary voltage, $4.0 \mathrm{kV}$; nebulizer pressure, $35 \mathrm{psig}$; fragmentor voltage, $100 \mathrm{~V}$ for NOR and IS; and collision energy, $15 \mathrm{eV}$ for NOR and $35 \mathrm{eV}$ for IS. Multiple-reaction monitoring used the transitions of $\mathrm{m} / \mathrm{z}$ $314 \rightarrow 265$ for NOR and $\mathrm{m} / \mathrm{z} 330 \rightarrow 181$ for IS.

\section{Intestinal lymphatic absorption}

Rats were divided randomly into two groups (five rats in each group), and fasted for $12 \mathrm{hrs}$ but granted free access 
to water prior to the experiment. One group was only orally administered with NOR-PC-SNEDDS (30 mg/kg). Another group was intraperitoneally injected with cycloheximide (CYC) (3 mg/kg) which was prepared with $0.9 \%$ physiological saline 90 mins before oral administration of NOR-PC-SNEDDS (30 mg/kg).

After administration, blood samples $(300 \mu \mathrm{L})$ were collected from the oculi chorioidea veins at $0,0.083$, $0.17,0.33,0.5,0.75,1,1.5,2,3,4,8,12$, and $24 \mathrm{hrs}$. The supernatant $(100 \mu \mathrm{L})$ was collected after centrifugation $\left(8000 \mathrm{rpm}, 4^{\circ} \mathrm{C}, 10 \mathrm{mins}\right)$ and stored at $-20^{\circ} \mathrm{C}$ until analysis.

The method of the sample pretreatment and the condition parameters of HPLC were the same as the previous experiment.

\section{Comparison of the metabolism of NOR-PC-SNEDDS and NOR in rat liver microsomes}

Rat liver microsomes were prepared by differential centrifugation. $^{20}$ The microsomal protein concentration was measured using a BCA protein assay kit.

The incubation mixture consisted of varying concentrations of NOR $(5,10,25$, or $50 \mu \mathrm{M})$ or NOR-PC-SNEDDS (calculated according to the percentage of NOR in NORPC-SNEDDS), rat liver microsomes $(0.5 \mathrm{mg}$ protein $/ \mathrm{mL})$, $2 \mathrm{mM}$ UDPGA, alamethicin $(25 \mu \mathrm{g} / \mathrm{mL}), 5 \mathrm{mM}$ D-saccharic acid 1,4-lactone, and $10 \mathrm{mM} \mathrm{MgCl}$. Then, the mixture was replenished with Tris- $\mathrm{HCl}$ buffer $(\mathrm{pH} 8.0)$ at a volume of $200 \mu \mathrm{L}$. After pre-incubation for $3 \mathrm{mins}$ at $37^{\circ} \mathrm{C}$, UDPGA was added to initiate the reaction. After incubation for $30 \mathrm{mins}, 1 \mathrm{~mL}$ ice ethyl acetate containing $10 \mu \mathrm{L}$ IS (sinomenine, $0.56 \mathrm{mM}$ ) was added to terminate the reaction. The mixture was vortex-shaking for $3 \mathrm{mins}$ and centrifuged at $12,000 \mathrm{rpm}$ for $10 \mathrm{mins}$. The supernatant $(800 \mu \mathrm{L})$ was transferred to a centrifuge tube and evaporated to dryness in a vacuum concentrator at $40^{\circ} \mathrm{C}$. The obtained residue was reconstituted in $100 \mu \mathrm{L} 0.1 \%$ formic acid water and centrifuged at $12,000 \mathrm{rpm}$ for $10 \mathrm{mins}$. The supernatant was used for HPLC analysis. The incubation system without UDPGA was used as a control group. All experiments were conducted in triplicate.

The effects of other components of NOR-PC-SNEDDS on the metabolism of NOR were further investigated. A quantity of $50 \mu \mathrm{M}$ NOR and a series of concentrations $(0.01,0.1,1 \mathrm{mg} / \mathrm{mL})$ of Cremophor EL, Labrasol, and Transcutol HP were added into the incubation system, respectively. The metabolic amount of NOR in every group was determined using HPLC after incubation for 30 mins. The NOR incubation group without the excipients was used as the negative control group and the metabolic amount of this group was set at $100 \%$ as a reference. The residual activity of the NOR in the Cremophor EL, Labrasol, and Transcutol HP groups was calculated, respectively.

The residue of NOR was determined using an Agilent 1260 liquid chromatography and a Shimpack CLC-ODS column $(150 \mathrm{~mm} \times 6 \mathrm{~mm}, 5 \mu \mathrm{m})$. The mobile phase consisting of methanol- $0.1 \%$ formic acid water $(15: 85, \mathrm{v} / \mathrm{v})$ was delivered at a flow rate of $1 \mathrm{~mL} / \mathrm{min}$. The column temperature was maintained at $40^{\circ} \mathrm{C}$. The injection volume was $20 \mu \mathrm{L}$, and the detection wavelength was set at $280 \mathrm{~nm}$.

\section{Statistical analysis}

PK Solver (Version 4; Thermo Fisher Scientific, Waltham, MA, USA) was used to calculate various pharmacokinetic parameters, including the maximum plasma concentration $\left(\mathrm{C}_{\max }\right)$, time to maximum plasma concentration $\left(\mathrm{T}_{\max }\right)$, area under the plasma concentration-time curve (AUC), and clearance rate $(\mathrm{Cl} / \mathrm{F})$ according to the non-compartment model. The experimental data were expressed as mean \pm SD and analyzed with statistical analysis software SPSS (SPSS Inc., Chicago, IL, USA). Statistical differences were assessed by one-way ANOVA in combination with independent-sample $t$-test. A value of $P<0.05$ was considered statistically significant.

\section{Results and discussion HPLC assay}

Representative chromatograms are shown in Figure 2. Under the chromatographic conditions described, the retention time of NOR was 7.8 mins. After fully validated, the method was successfully applied to determine the concentration of NOR in NOR-PC.

\section{Characterization of NOR-PC}

DSC was used to study the possible interaction between NOR and phospholipids. NOR had two endothermic peaks at $132^{\circ} \mathrm{C}$ and $194^{\circ} \mathrm{C}$ as shown in Figure 3D. Thermogram of phospholipids (Figure 3C) showed a sharp endothermic peak at $236^{\circ} \mathrm{C}$ which was the transition from gel state to liquid crystal state. This might be the melting of phospholipid hydrocarbon chain, isomerization, or crystal form 


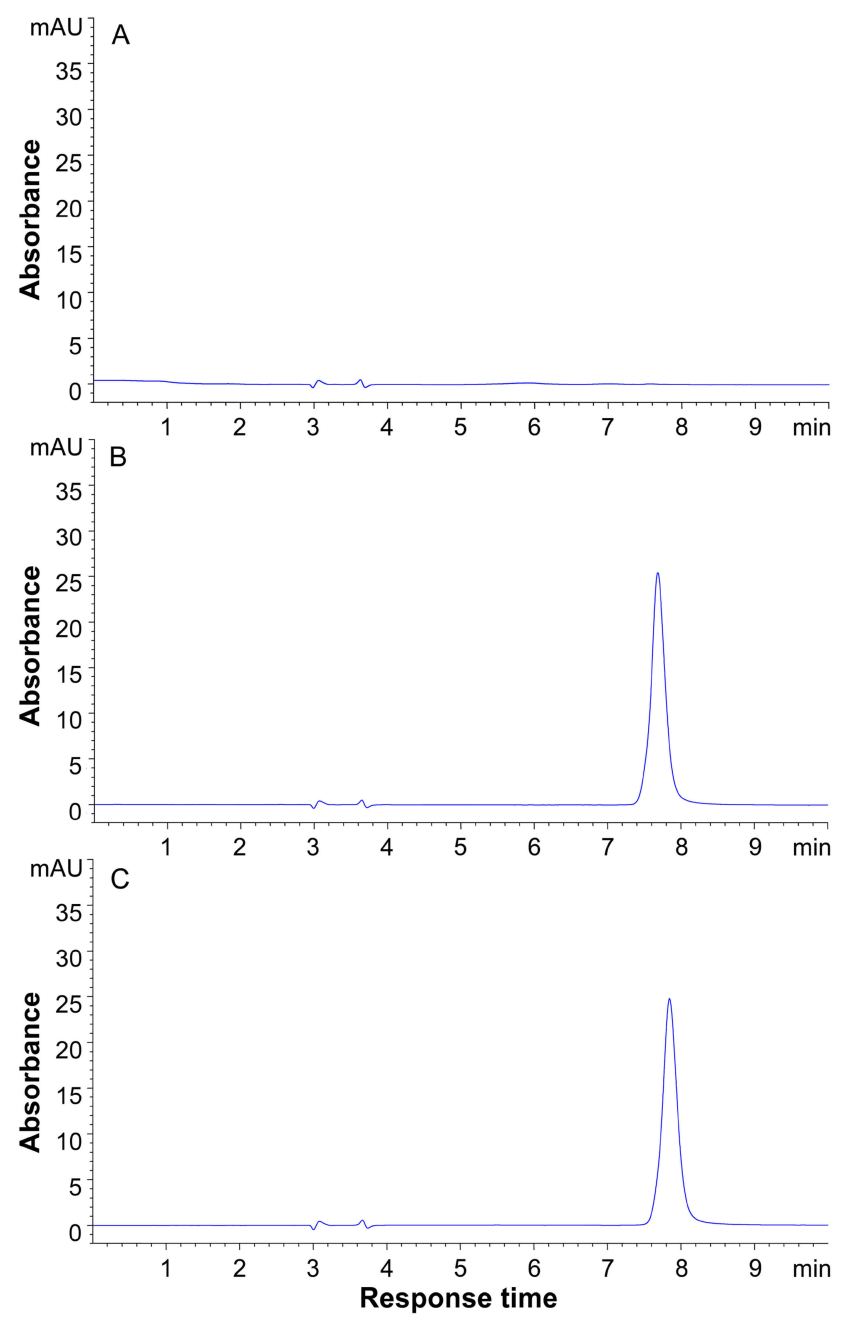

Figure 2 HPLC chromatograms of NOR.

Notes: (A) Blank sample. (B) Blank sample spike with NOR. (C) NOR-PC. Abbreviations: NOR, norisoboldine; NOR-PC, norisoboldine-phospholipid complex.

change. ${ }^{18}$ Both the peaks of NOR and phospholipid disappeared in the DSC curve of NOR-PC (Figure 3A). The result indicated that NOR was dissolved completely in the soybean phospholipid and the interactions between NOR and phospholipid occurred. The interactions between NOR and PC contributed to entrapment of the NOR into the drug-loading system and formation of the NOR-PC. Thermogram of the mixture of NOR and phospholipids showed an endothermic peak of NOR-PC $160^{\circ} \mathrm{C}$ and an endothermic peak of NOR $135^{\circ} \mathrm{C}$ (Figure 3B), which might be due to the melting of soybean phospholipids and the interaction with the NOR during the heating process.

In the phospholipid FT-IR spectrum (Figure 4C), 1235.8 $\mathrm{cm}^{-1}$ was the stretching vibration peak of $\mathrm{P}=\mathrm{O}, 1087.8 \mathrm{~cm}^{-1}$ was the stretching vibration peak of P-O-C, and $968.3 \mathrm{~cm}^{-1}$

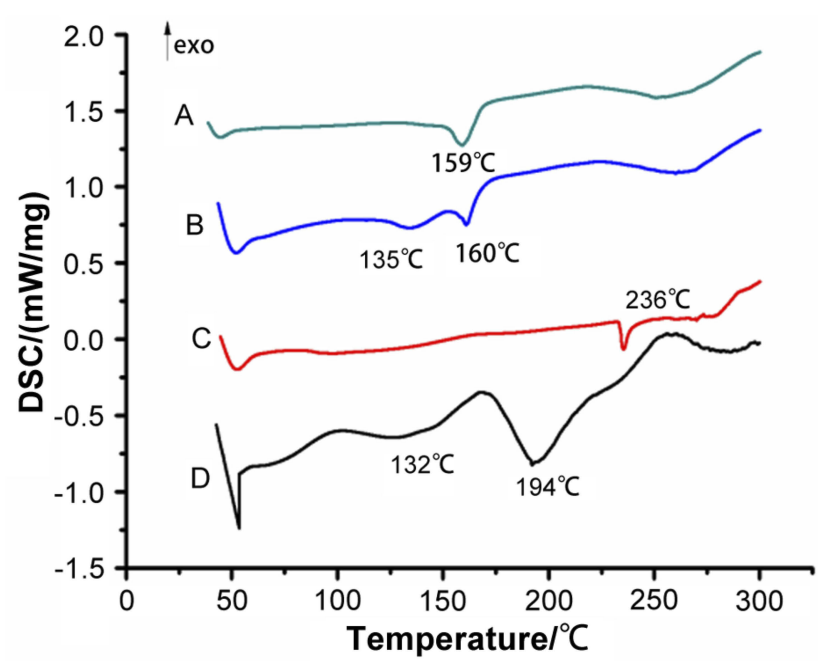

Figure 3 DSC thermograms.

Notes: (A) NOR-PC. (B) Physical mixture of NOR and phospholipids. (C) Phospholipids. (D). NOR.

Abbreviations: DSC, differential scanning calorimetry; NOR-PC, norisoboldinephospholipid complex; NOR, norisoboldine.

was the stretching vibration peak of $\mathrm{N}^{+}(\mathrm{CH} 3)_{3} \cdot{ }^{21,22}$ FT-IR spectrum of the physical mixture of NOR and phospholipids (Figure 4B) was in accordance with NOR-PC (Figure 4A) which was almost the superposition of the FT-IR spectrum of the NOR (Figure 4D) and phospholipids. The main absorption peaks of NOR and phospholipid were observed. Compared with the FT-IR spectrums of NOR and phospholipid, both absorption peaks of NOR at $1586.7 \mathrm{~cm}^{-1}$ (for $\mathrm{C}=\mathrm{C}$ stretch vibration) and phospholipids at $968.3 \mathrm{~cm}^{-1}$ (for $\mathrm{N}^{+}(\mathrm{CH} 3)_{3}$ stretch vibration) were reduced. There might be hydrogen bonds or van der Waals forces between NOR and

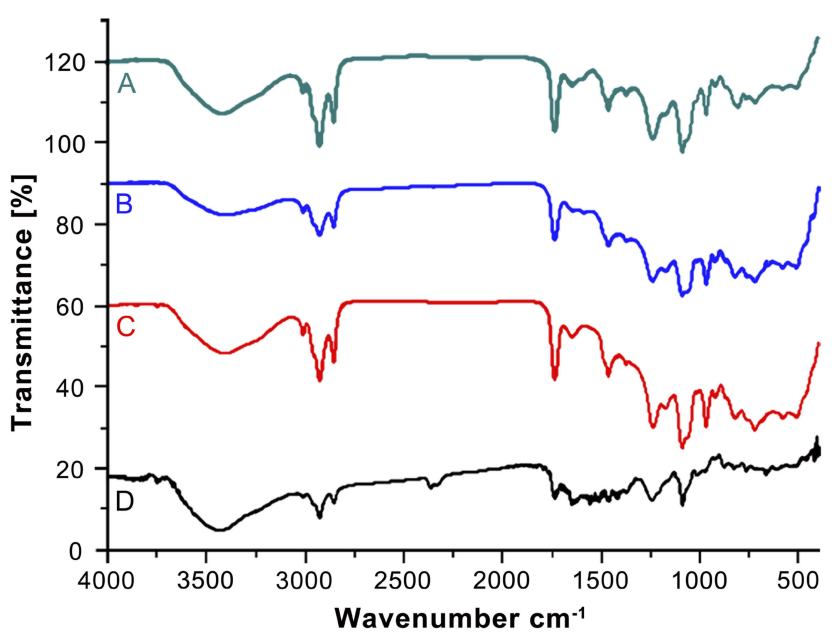

Figure 4 Infrared spectra.

Notes: (A) NOR-PC. (B) Physical mixture of NOR and phospholipids. (C) Phospholipids. (D). NOR.

Abbreviations: NOR-PC, norisoboldine-phospholipid complex; NOR, norisoboldine. 
soybean phospholipids. Due to the presence of hydroxyl groups on the benzene ring of the drug, hydrogen bonds or van der Waals forces may form between the drug and the phospholipid, which make it easier to be embedded in the drug delivery system.

\section{Solubility studies}

The apparent solubilities of NOR, the physical mixture of NOR, and phospholipid as well as NOR-PC in double distilled water and n-octanol are shown in Table 1, respectively. The liposolubility of NOR in double distilled water and n-octanol increased significantly after the preparation of NOR-PC.

\section{Selection of oil phase, surfactant, and cosurfactant}

The solubilities of NOR and NOR-PC in various oil phases, surfactants, and cosurfactants are shown in Figure 5. The solubility of NOR-PC was higher than NOR in various carriers. Among all kinds of oil phases and cosurfactants, the solubility of NOR-PC was the highest in Capryol 90 and Transcutol HP. Therefore, Capryol 90 and Transcutol HP were chosen as the oil phase and the cosurfactant to make the prescription have a larger drug loading. As shown in Figure 5, Labrasol had the highest solubility among surfactants, but its emulsifying ability was weak. Cremophor EL had the strongest emulsifying ability among surfactants. Based on pre-experiment results, the ratio of Labrasol and Cremophor EL to be 3:2 was the best of all ratios. Therefore, Labrasol: Cremophor EL (3:2) was chosen as surfactant to ensure a larger drug loading and stronger emulsifying ability. However, when Capryol 90, Labrasol:Cremophor EL (3:2), and Transcutol HP were selected as the oil, surfactant, and cosurfactant, which was difficult to emulsify, and large oil droplets were observed on the liquid surface.

Table I Solubilities of NOR, physical mixture, and NOR-PC in water and n-octanol

\begin{tabular}{|l|l|l|}
\hline \multirow{2}{*}{ Sample } & \multicolumn{2}{|l|}{ Solubility $(\mathrm{mg} / \mathrm{mL})$} \\
\cline { 2 - 3 } & Double distilled water & n-octanol \\
\hline NOR & $0.38 \pm 0.03$ & $0.48 \pm 0.03$ \\
Physical mixture & $0.7 I \pm 0.03$ & $0.84 \pm 0.02$ \\
NOR-PC & $0.84 \pm 0.02 * *$ & $1.02 \pm 0.03 * *$ \\
\hline
\end{tabular}

Notes: Data are expressed as mean $\pm S D(n=3) . * * p<0.01$ compared with NOR group.

Abbreviations: NOR, norisoboldine; NOR-PC, norisoboldine-phospholipid complex.

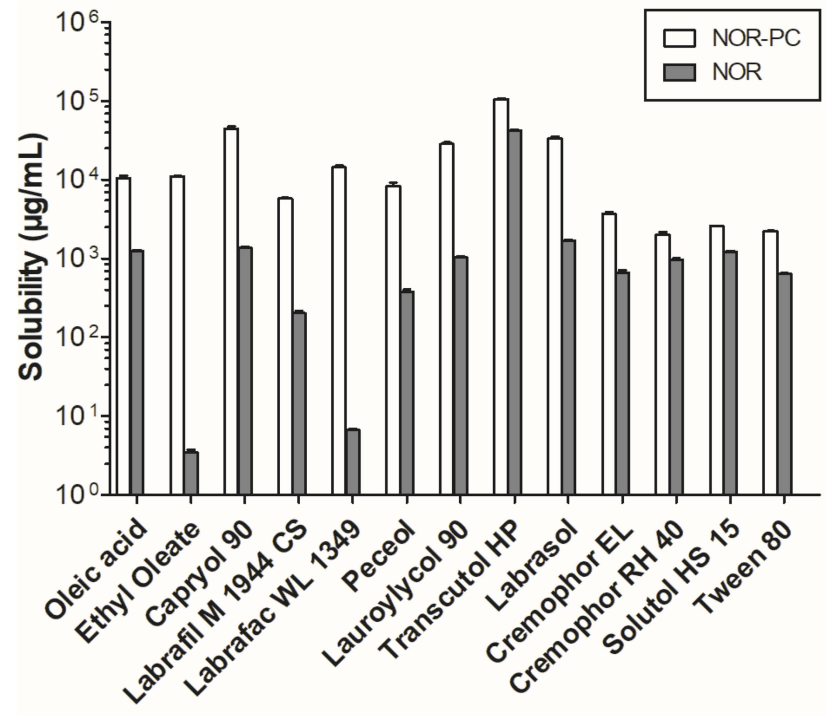

Figure 5 Solubility of NOR-PC and NOR in various carriers. Abbreviations: NOR-PC, norisoboldine-phospholipid complex; NOR, norisoboldine.

Therefore, the oil phase was altered to Ethyl oleate to improve self-emulsification. Finally, Ethyl oleate, Labrasol:Cremophor EL (3:2), and Transcutol HP were selected as the oil, surfactant, and cosurfactant, respectively.

\section{Construction of pseudo-ternary phase diagram}

The pseudo-ternary phase diagram can be used to screen the appropriate prescription ratio and drug loading to get the balance between drug loading and self-emulsifying efficiency in the preparation of SNEDDS. Ethyl oleate, Labrasol:Cremophor EL (3:2), and Transcutol HP were selected as the oil, surfactant, and cosurfactant, respectively. The oil phase, surfactant, and cosurfactant were used as the three vertices of the pseudo-ternary phase diagrams, respectively. Origin 8.0 software was used to get blank pseudoternary phase diagram. The NOR-PC was added to the blank prescription one by one. Finally, the content of NOR was $1 \%$ and the drug-loaded pseudo-ternary phase diagram is shown in Figure 6. The final formulation of NOR-PC-SNEDDS was NOR-PC/Ethyl oleate/Labrasol/Cremophor EL/Transcutol $\mathrm{HP}=1: 2: 3.36: 2.24: 2.4(\mathrm{w} / \mathrm{w} / \mathrm{w} / \mathrm{w})$.

\section{Characterization of NOR-PC-SNEDDS}

After dilution with distilled water at a ratio of 1:50 (w/w), the particle size, polydispersity coefficient, and zeta potential of NOR-PC-SNEDDS were $36.72 \pm 1.47 \mathrm{~nm}, 0.09$ \pm 0.02 , and $-4.91 \pm 0.49 \mathrm{mV}$, respectively. The particle size 


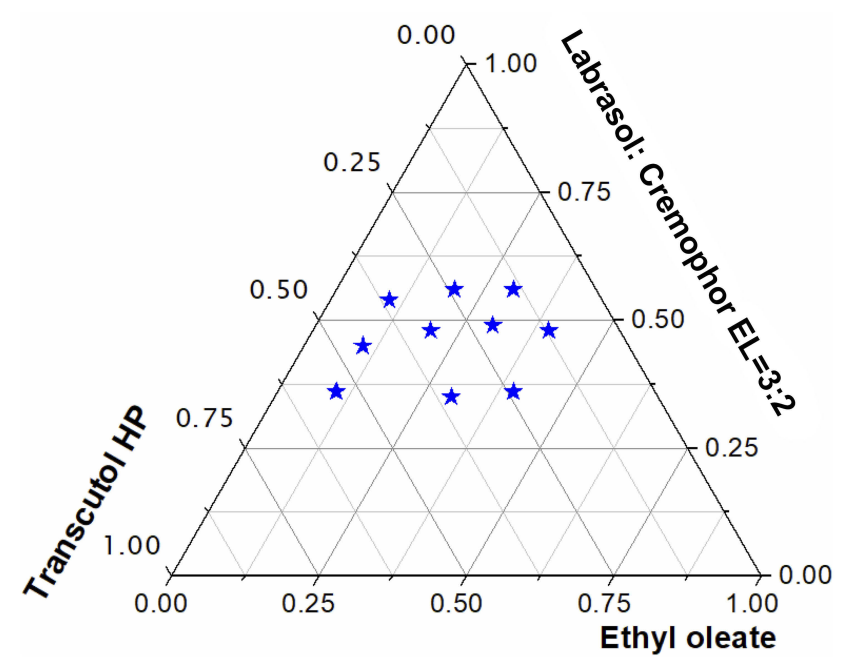

Figure 6 Pseudo-ternary phase diagram of the SNEDDS formulation composed of Ethyl oleate, Transcutol HP, Labrasol:Cremophor EL (3:2), and NOR-PC.

Abbreviations: SNEDDS, self-nanoemulsifying drug delivery system; NOR-PC, norisoboldine-phospholipid complex.

and zeta potential of the emulsion droplets are important indicators for evaluating the self-emulsification properties because they determine the rate and extent of drug release as well as the stability of the emulsion. Studies have shown that small particle size is beneficial for bioavailability. $^{23,24}$ In addition, the zeta potential also affects the stability of SNEDDS. High zeta potential is conducive to system stability. ${ }^{25}$
The transmission electron micrograph of NOR-PCSNEDDS is shown in Figure 7. It could be seen that NOR-PC-SNEDDS dispersed uniformly in aqueous solution and in globular form with a narrow particle size change.

\section{Thermodynamic stability of NOR-PC-SNEDDS}

The effects of different media, dilution factors, and freeze-thaw on particle size and PDI are shown in Table 2. The particle size and PDI of NOR-PC-SNEDDS were stable after dilution by 50, 250, and 1000 times in double distilled water, $0.1 \mathrm{M} \mathrm{HCl}$ solution $(\mathrm{pH} 1.2)$ and phosphate buffer solution ( $\mathrm{pH}$ 6.8), respectively. Moreover, the particle size and PDI were stable after repeated freezing and thawing for three times.

\section{Pharmacokinetic studies}

The pharmacokinetic characteristics of NOR in rats after oral administration of NOR, NOR-PC, NOR-PC-SNEDDS, or intravenous injection of NOR were compared. The plasma concentration-time curves of NOR in four groups are shown in Figure 8. The main pharmacokinetic parameters and the absolute bioavailability of NOR are listed in Table 3 .

The results showed that the pharmacokinetic parameters of NOR-PC-SNEDDS group were significantly different

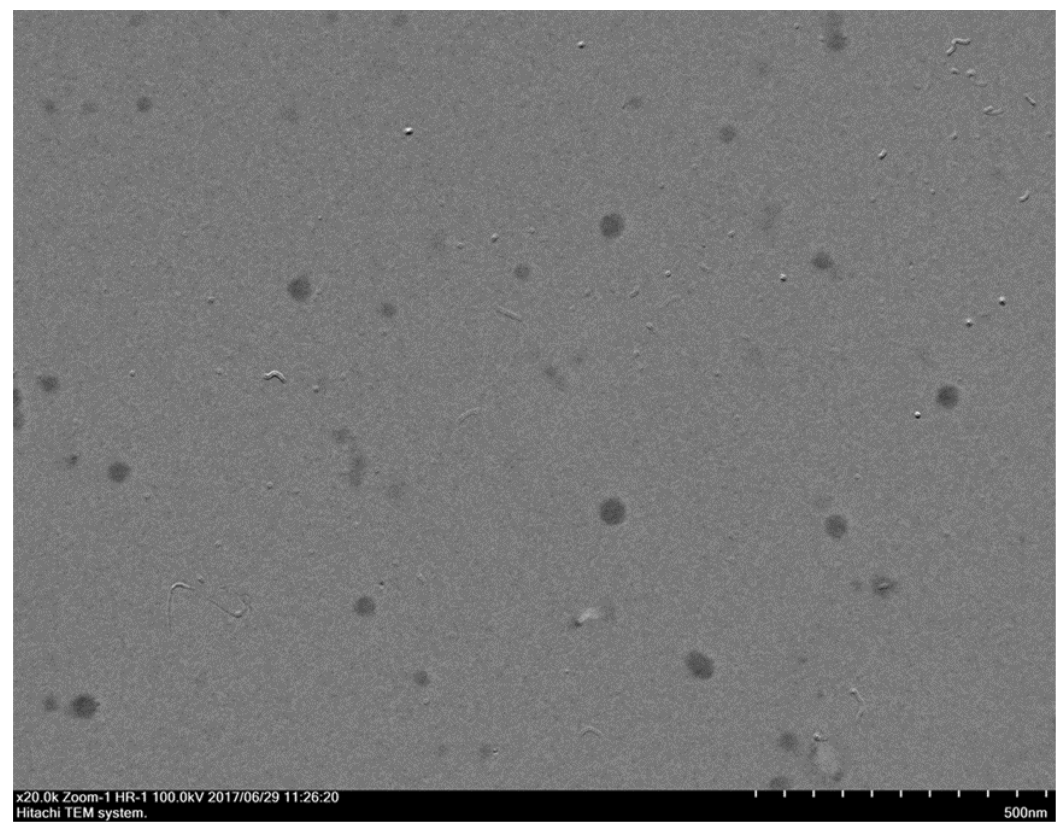

Figure 7 Transmission electron microscope image of NOR-PC-SNEDDS formulation $(\times 20,000$ magnification).

Abbreviations: SNEDDS, self-nanoemulsifying drug delivery system; NOR-PC, norisoboldine-phospholipid complex. 
Table 2 Thermodynamic stability of NOR-PC-SNEDDS

\begin{tabular}{|l|l|l|l|}
\hline & $\begin{array}{l}\text { Dilution } \\
\text { factor }\end{array}$ & $\begin{array}{l}\text { Particle } \\
\text { size } \mathbf{( n m})\end{array}$ & $\begin{array}{l}\text { Polydispersity } \\
\text { index }\end{array}$ \\
\hline $\begin{array}{l}\text { Double distilled } \\
\text { water }\end{array}$ & 50 & $36.72 \pm 1.47$ & $0.09 \pm 0.02$ \\
& 250 & $36.02 \pm 1.04$ & $0.08 \pm 0.01$ \\
& 1000 & $36.46 \pm 2.53$ & $0.07 \pm 0.01$ \\
$\mathrm{pH} 6.8$ & 50 & $33.62 \pm 2.91$ & $0.10 \pm 0.02$ \\
& 250 & $36.69 \pm 2.1 \mathrm{I}$ & $0.08 \pm 0.00$ \\
& 1000 & $36.22 \pm 1.12$ & $0.07 \pm 0.02$ \\
$\mathrm{pH} \mathrm{I.2}$ & 50 & $34.68 \pm 1.28$ & $0.07 \pm 0.01$ \\
& 250 & $36.1 \mathrm{I} \pm 1.05$ & $0.07 \pm 0.02$ \\
& 1000 & $35.43 \pm 1.20$ & $0.07 \pm 0.00$ \\
After heating-cooling & 250 & $36.59 \pm 1.04$ & $0.08 \pm 0.01$ \\
test & & & \\
\hline
\end{tabular}

Note: Data are expressed as mean $\pm \operatorname{SD}(n=3)$.

Abbreviations: SNEDDS, self-nanoemulsifying drug delivery system; NOR-PC, norisoboldine-phospholipid complex.
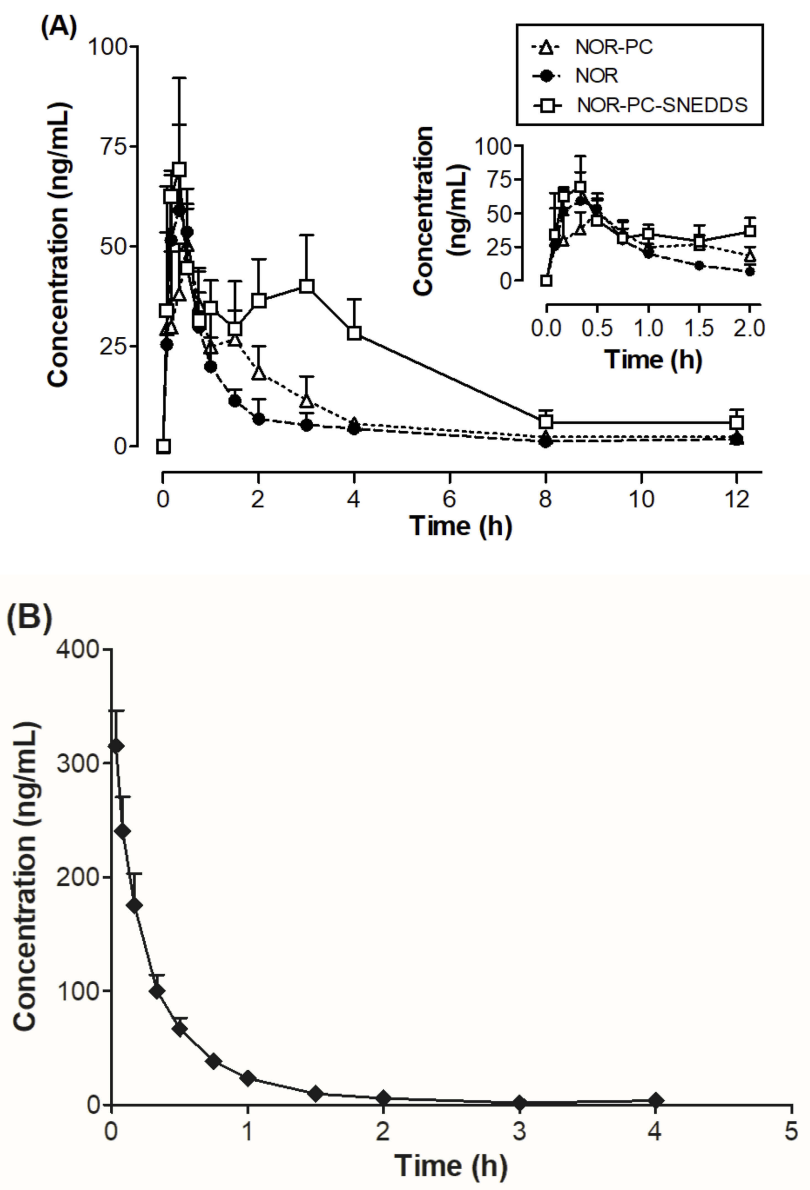

Figure 8 (A) Plasma concentration-time profiles of NOR after oral administration of NOR, NOR-PC, and NOR-PC-SNEDDS formulation at a dose of $30 \mathrm{mg} / \mathrm{kg}$ (mean $\pm S D, n=5)$; (B) Plasma concentration-time profiles of NOR after a tail vein injection at a dose of $2 \mathrm{mg} / \mathrm{kg}($ mean $\pm \mathrm{SD}, \mathrm{n}=5)$.

Abbreviations: SNEDDS, self-nanoemulsifying drug delivery system; NOR-PC: norisoboldine-phospholipid complex; NOR, norisoboldine. from those of NOR and NOR-PC groups. Compared with rats in NOR group, rats in NOR-PC-SNEDDS group showed an increase of 2.67, 3.67, and 3.72 folds in $\mathrm{T}_{1 / 2}$, $\mathrm{AUC}_{0-\mathrm{t}}$, and $\mathrm{AUC}_{0-\infty}$ values, and a decrease of $\mathrm{Cl} / \mathrm{F}$ value to $27.78 \%$. Similarly, compared with NOR-PC group, the $\mathrm{T}_{1 / 2}$, $\mathrm{AUC}_{0-\mathrm{t}}$, and $\mathrm{AUC}_{0-\infty}$ values in rats of NOR-PC-SNEDDS group were increased to $2.29,2.73$, and 2.75 folds, and the $\mathrm{Cl} / \mathrm{F}$ decreased to $37.04 \%$. Furthermore, the absolute bioavailability of NOR in the NOR-PC-SNEDDS group was found to be significantly increased and the value was $372 \%$ and $275 \%$ in relative to NOR group and NOR-PC group, respectively. However, the pharmacokinetic parameters in NOR-PC group were not markedly different from those of the NOR group. The results implied that certain compositions in excipients of NOR-PC-SNEDDS might improve the bioavailability of NOR rather than phospholipid.

\section{Intestinal lymphatic absorption}

The plasma concentration-time curves of NOR-PCSNEDDS group and NOR-PC-SNEDDS-CYC group are illustrated in Figure 9. The main pharmacokinetic parameters are summarized in Table 4.

The pharmacokinetic parameters of the NOR-PCSNEDDS-CYC group were significantly different from those of NOR-PC-SNEDDS group. Compared with NOR-PC-SNEDDS group, the $\mathrm{T}_{\max }, \mathrm{C}_{\max }, \mathrm{AUC}_{0-\mathrm{t}}$, and $\mathrm{AUC}_{0-\infty}$ values of NOR-PC-SNEDDS-CYC group were found to be significantly decreased to $29.63 \%, 29.61 \%$, $49.04 \%$, and $60.53 \%$, respectively. Meanwhile, $\mathrm{Cl} / \mathrm{F}$ value for the NOR-PC-SNEDDS-CYC group increased to 1.7 folds of that of the NOR-PC-SNEDDS group.

The intestinal lymphatic transport is an important way to enhance the absorption of lipophilic drugs. To investigate the lymphatic transport of drugs, a simplified and effective in vivo model named chylomicron flow blocking approach was commonly carried out. ${ }^{26}$ Chylomicrons are very large, spherical particles, and mainly composed of triglyceride (85-92\%). The chylomicrons are formed and secreted by Golgi apparatus and selectively enter the lymph vessel. Drugs entering the lymphatic system need to bind to triglycerides in the chylomicrons. ${ }^{27}$ Cycloheximide, a non-specific protein synthesis inhibitor that inhibits the secretion of chylomicron from the enterocyte, can block the lymphatic transport and therefore result in the absorption decrease of drugs. In the present study, the results demonstrated that cycloheximide blocked the lymphatic transport of SNEDDS formulation, thereby reduced the absorption of SNEDDS formulation. 
Table 3 Bioavailability and pharmacokinetic parameters of NOR after oral administration of NOR, NOR-PC, NOR-PC-SNEDDS, and tail vein injection of NOR

\begin{tabular}{|l|l|l|l|l|}
\hline Parameter & NOR-IV & NOR & NOR-PC & NOR-PC-SNEDDS \\
\hline $\mathrm{T}_{1 / 2}(\mathrm{~h})$ & $0.55 \pm 0.08$ & $2.50 \pm 0.40$ & $2.92 \pm 0.70$ & $6.68 \pm 1.86^{* * \#}$ \\
$\mathrm{~T}_{\max }(\mathrm{h})$ & $0.03 \pm 0$ & $0.33 \pm 0.17$ & $0.42 \pm 0.19$ & $0.27 \pm 0.09$ \\
$\mathrm{C}_{\max }(\mathrm{ng} / \mathrm{mL})$ & $315.26 \pm 31.08$ & $65.42 \pm 18.09$ & $61.01 \pm 17.88$ & $74.97 \pm 15.66$ \\
$\mathrm{AUC} \mathrm{C}_{0-\mathrm{t}}(\mathrm{ng} / \mathrm{mL} * \mathrm{~h})$ & $119.46 \pm 9.37$ & $80.25 \pm 18.74$ & $107.84 \pm 21.82$ & $294.12 \pm 77.80^{* * \#}$ \\
$\mathrm{AUC} \mathrm{C}_{-\infty}\left(\mathrm{ng} / \mathrm{mL}^{*} \mathrm{~h}\right)$ & $122.85 \pm 12.38$ & $86.98 \pm 19.33$ & $117.83 \pm 33.90$ & $324.00 \pm 104.48^{* * \#}$ \\
$\mathrm{Cl} / \mathrm{F}(\mathrm{L} / \mathrm{h} / \mathrm{g})$ & $0.02 \pm 0.002$ & $0.36 \pm 0.10$ & $0.27 \pm 0.06$ & $0.10 \pm 0.03^{* * \#}$ \\
$\mathrm{~F}(\%)$ & - & 4.72 & 6.39 & 17.58 \\
\hline
\end{tabular}

Notes: Data are expressed as mean $\pm S D(n=5)$. ${ }^{*} * P<0.01$ compared with NOR group; ${ }^{\#} P<0.01$ compared with NOR-PC group.

Abbreviations: NOR, norisoboldine; NOR-PC, norisoboldine-phospholipid complex; SNEDDS, self-nanoemulsifying drug delivery system; $\mathrm{T}_{1 / 2}$, half-life elimination time; $T_{\max }$, time to maximum plasma concentration; $C_{\max }$, maximum plasma concentration; $A U C$, the area under the plasma concentration-time curve; $F$, relative bioavailability.

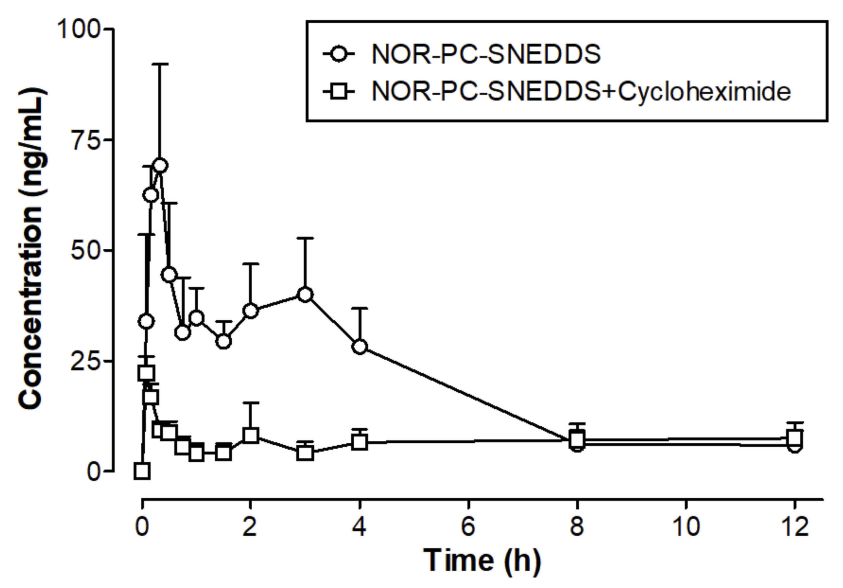

Figure 9 Blood concentration-time curves of intraperitoneal injection of cycloheximide in rats by intragastric administration of NOR-PC-SNEDDS and intragastric administration of NOR-PC-SNEDDS.

Abbreviations: SNEDDS, self-nanoemulsifying drug delivery system; NOR-PC, norisoboldine-phospholipid complex.

Table 4 Pharmacokinetic parameters of NOR-PC-SNEDDS group and NOR-PC-SNEDDS-CYC group

\begin{tabular}{|l|l|l|}
\hline Parameter & $\begin{array}{l}\text { NOR-PC- } \\
\text { SNEDDS }\end{array}$ & $\begin{array}{l}\text { NOR-PC-SNEDDS- } \\
\text { CYC }\end{array}$ \\
\hline $\mathrm{T}_{1 / 2}(\mathrm{~h})$ & $6.68 \pm 1.86$ & $12.91 \pm 4.64$ \\
$\mathrm{~T}_{\max }(\mathrm{h})$ & $0.27 \pm 0.09$ & $0.08 \pm 0 *$ \\
$\mathrm{C}_{\max }(\mathrm{ng} / \mathrm{mL})$ & $74.97 \pm 15.66$ & $22.20 \pm 3.8 \mathrm{I}^{* *}$ \\
$\mathrm{AUC}_{0-\mathrm{t}}(\mathrm{ng} / \mathrm{mL} \times \mathrm{h})$ & $294.12 \pm 77.80$ & $144.24 \pm 59.76^{* *}$ \\
$\mathrm{AUC}_{0-\infty}(\mathrm{ng} / \mathrm{mL} \times \mathrm{h})$ & $324.00 \pm 104.48$ & $196.12 \pm 62.37^{*}$ \\
$\mathrm{Cl} / \mathrm{F}(\mathrm{L} / \mathrm{h} / \mathrm{g})$ & $0.10 \pm 0.03$ & $0.17 \pm 0.05^{*}$ \\
\hline
\end{tabular}

Notes: Data are expressed as mean $\pm S D(n=5)$. $* P<0.05$ compared with NOR-PCSNEDDS group; $* * P<0.01$ compared with NOR-PC-SNEDDS group.

Abbreviations: SNEDDS, self-nanoemulsifying drug delivery system; NOR-PC, norisoboldine-phospholipid complex; CYC, cycloheximide.

It has been reported that unsaturated long-chain fatty acids, such as oleic acid, peceol, maisine $35-1$, have the ability to enhance the systemic bioavailability by the intestinal lymphatic transport. ${ }^{28}$ Therefore, Ethyl oleate in NOR-PC-SNEDDS prescription might contribute to promote the absorption of NOR in NOR-PC-SNEDDS via lymphatic transport and increase the bioavailability.

\section{Comparison of the metabolism of NOR- PC-SNEDDS and NOR in rat liver microsomes}

As shown in Figure 10, the metabolic rates of NOR in the series of concentrations of NOR-PC-SNEDDS groups decreased compared with NOR group. The difference was more significant following the increase of the concentration of NOR.

The effect of components of NOR-PC-SNEDDS on the metabolism of NOR Phase II was further investigated. The inhibition of Cremophor EL, Labrasol, and Transcutol HP on NOR Phase II metabolism is shown in Figure 11. Transcutol HP had no significant effect on NOR Phase II metabolism. However, Cremophor EL and Labrasol had a certain inhibitory effect on NOR Phase II metabolism and the inhibition was more obvious with concentration increasing.

UGTs (UDP-glucuronosyltransferases) are important Phase II metabolic enzymes in organisms. UGTs can bind glucuronic acid on the functional groups of compounds, increase water solubility of compounds, make compounds lose biological activity and elimination from the body. Previous study has shown that NOR was mainly metabolized to NOR-9-O-glucuronide by UGTs. ${ }^{29}$ Therefore, our results indicated the excipient components of NOR-PC-SNEDDS reduce the metabolism of NOR and improve the bioavailability. 


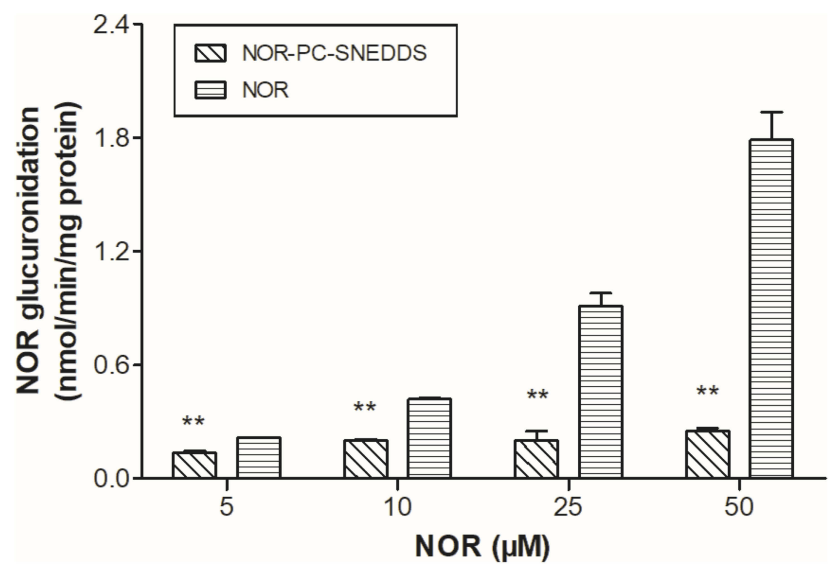

Figure 10 Comparison of the metabolism of NOR-PC-SNEDDS and NOR in rat liver microsomes.

Note: $* * P<0.01$, there was a very significant difference compared to the NOR group. Abbreviations: SNEDDS, self-nanoemulsifying drug delivery system; NOR-PC, norisoboldine-phospholipid complex; NOR, norisoboldine.

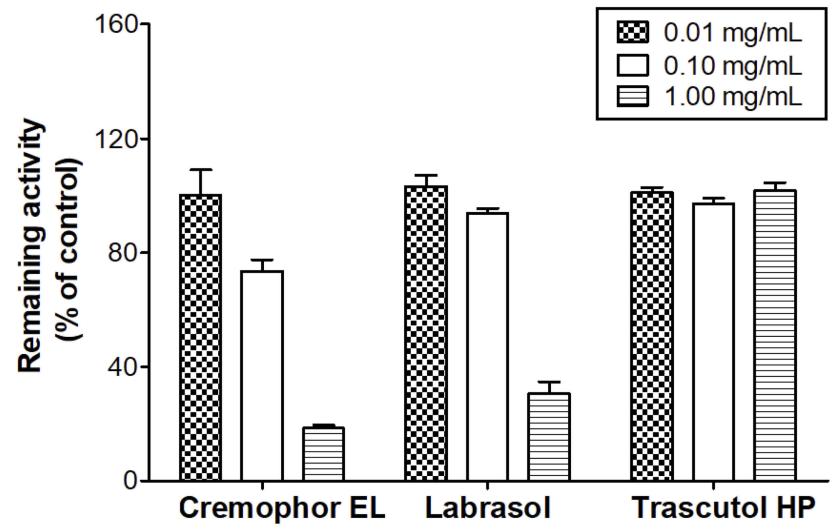

Figure II Effects of composition of NOR-PC-SNEDDS on NOR Phase II metabolism.

Abbreviations: SNEDDS, self-nanoemulsifying drug delivery system; NOR-PC, norisoboldine-phospholipid complex; NOR, norisoboldine.

\section{Conclusion}

This study designed and evaluated a novel NOR-PCSNEDDS which improved the oral bioavailability of NOR. NOR-PC-SNEDDS functions through enhancing intestinal lymphatic transport and inhibiting the liver metabolism of NOR. The findings in this paper provide a new idea for the further development and application of NOR.

\section{Acknowledgment}

This work was supported by the China National Natural Science Foundation (No. 81872881; 81373426) and the "Double First-Class" University project (CPU2018GY10).

\section{Disclosure}

The authors report no conflicts of interest in this work.

\section{References}

1. Scott DL, Wolfe F, Huizinga TW. Rheumatoid arthritis. Lancet. 2010;376:1094-1108. doi:10.1016/S0140-6736(10)60826-4

2. Zeng XF, Zhu SI, Tan XP, et al. Disease burden and quality of life of rheumatoid arthritis in China: a systematic review. Chin J Evid Based Med. 2013;13:300-307.

3. Chou GX, Norio N, Ma CM, et al. Isoquinoline alkaloids from Lindera aggregate. Chin J Nat Med. 2005;03(5):272-275.

4. Luo Y, Liu M, Xia Y, et al. Therapeutic effect of norisoboldine, an alkaloid isolated from Radix Linderae, on collagen-induced arthritis in mice. Phytomedicine. 2010;17(10):726-731. doi:10.1016/j. phymed.2010.01.013

5. Wei ZF, Jiao XL, Wang T, et al. Norisoboldine alleviates joint destruction in rats with adjuvant-induced arthritis by reducing RANKL, IL-6, PGE (2), and MMP-13 expression. Acta Pharmacol Sin. 2013;34(3):403-413. doi:10.1038/aps.2012.187

6. Tong B, Dou Y, Wang T, et al. Norisoboldine ameliorates collagen-induced arthritis through regulating the balance between Th17 and regulatory $\mathrm{T}$ cells in gut-associated lymphoid tissues. Toxicol Appl Pharmacol. 2015;282(1):90-99. doi:10.1016/j.taap. 2014.11.008

7. Lu Q, Lu S, Gao XH, et al. Norisoboldine, an alkaloid compound isolated from Radix Linderae, inhibits synovial angiogenesis in adjuvant-induced arthritis rats by moderating Notch1 pathway-related endothelial tip cell phenotype. Exp Biol Med. 2012;237:919-932. doi:10.1258/ebm.2012.011416

8. Lu Q, Tong B, Luo YB, et al. Norisoboldine suppresses VEGF-induced endothelial cell migration via the cAMP-PKA-NF-kB/Notch1 pathway. PLoS One. 2013;8:12. doi:10.1371/journal.pone.0081220

9. Chen JZ, Chou GX, Wang CH, et al. Pharmacokinetics and bioavailability of norisoboldine and its metabolite in Rats. Chin JMAP. 2012;29(6):473-477.

10. Eltobshi AA, Mohamed EA, Abdelghani GM, et al. Self-nanoemulsifying drug-delivery systems for potentiated anti-inflammatory activity of diacerein. Int $J$ Nanomedicine. 2018;13(1):5887-5907. doi:10.2147/IJN.S174146

11. Salem HF, Kharshoum RM, Saved OM, et al. Formulation development of self-nanoemulsifying drug delivery system of celecoxib for the management of oral cavity inflammation. $J$ Liposome Res. 2018;23:1-11.

12. Baldi A, Chaudhary M, Sethi S, et al. Armamentarium of nanoscaled lipid drug delivery systems customized for oral administration: in silico docking patronage, absorption pheonmenon, preclinical status, clinical status and future prospects. Colloids Surf B Biointerfaces. 2018;170:637-647. doi:10.1016/j.colsurfb.2018.06.061

13. Buyukozturk F, Benneyan JC, Carrier RL. Impact of emulsionbased drug delivery systems on intestinal permeability and drug release kinetics. J Control Release. 2010;142(1):22-30. doi:10.10 16/j.jconrel.2009.10.005

14. Mamadou G, Charrueau C, Dairou J, et al. Increased intestinal permeation and modulation of presystemic metabolism of resveratrol formulated into self-emulsifying drug delivery systems. Int J Pharm. 2017;521(1-2):150-155. doi:10.1016/j.ijpharm.2017.02.036

15. Valicherla GR, Dave KM, Syed AA, et al. Formulation optimization of Docetaxel loaded self-emulsifying drug delivery system to enhance bioavailability and anti-tumor activity. Sci Rep. 2016;6:26895. doi:10.1038/srep26895

16. Shen JY, Bi JP, Tian HL, et al. Preparation and evaluation of a selfnanoemusifying drug delivery system loaded with akebia saponin Dphospholipid complex. Int J Nanomedicine. 2016;11:4919-4929. doi:10.2147/IJN.S108765

17. Zhang J, Peng Q, Shi S, et al. Preparation, charaterization, and in vivo evaluation of a self-nanoemulsifying drug delivery system (SNEDDS) loaded with morin-phospholipid complex. Int $J$ Nanomedicine. 2011;6:3405-3414. doi:10.2147/IJN.S25824 
18. Xiao YY, Song YM, Chen ZP, et al. The preparation of silybinphospholipid complex and the study on its pharmacokinetics in rats. Int J Pharm. 2006;307(1):77-82. doi:10.1016/j.ijpharm.2005.10.001

19. Manconi M, Nácher A, Merino V, et al. Improving oral bioavailability and pharmacokinetics of liposomal metformin by glycerolphosphate-chitosan microcomplexation. AAPS PharmSciTech. 2013;14 (2):485-496. doi:10.1208/s12249-013-9926-4

20. Eriksson LC. Preparation of liver microsomes with high recovery of endoplasmic reticulum and a low grade of contamination. Biochim Biophys Acta. 1978;508(1):155-164. doi:10.1016/0005-2736(78)90197-9

21. Jena SK, Singh C, Dora CP, et al. Development of tamoxifen-phospholipid complex: novel approach for improving solubility and bioavailability. Int J Pharm. 2014;473(1-2):1-9. doi:10.1016/j.ijpharm.2014.06.056

22. Hou Z, Li Y, Huang Y, et al. Phytosomes loaded with mitomycin Csoybean phosphatidylcholine complex developed for drug delivery. Mol Pharm. 2013;10(1):90-101. doi:10.1021/mp300489p

23. Larsen AT, Ohlsson AG, Polentarutti B, et al. Oral bioavailability of cinnarizine in dogs: relation to SNEDDS droplet size, drug solubility and in vitro precipitation. Eur J Pharm Sci. 2013;48(1-2):339-350. doi:10.1016/j.ejps.2012.11.004

24. Nielsen FS, Petersen KB, Mullertz A. Bioavailability of probucol from lipid and surfactant based formulations in minipigs: influence of droplet size and dietary state. Eur J Pharm Biopharm. 2008;69 (2):553-562. doi:10.1016/j.ejpb.2007.12.020
25. Date AA, Desai N, Dixit R, et al. Self-nanoemulsifying drug delivery systems: formulation insights, applications and advances. Nanomedicine (Lond). 2010;5(10):1595-1616. doi:10.2217/nnm.10.126

26. Sun $M$, Zhai $X$, Xue $K$, et al. Intestinal absorption and intestinal lymphatic transport of sirolimus from self-microemulsifying drug delivery systems assessed using the single-pass intestinal perfusion (SPIP) technique and a chylomicron flow blocking approach: linear correlation with oral bioavailabilities in rats. Eur J Pharm Sci. 2011;43(3):132-140. doi:10.1016/j.ejps.2011.04.011

27. Dahan A, Hoffman A. Evaluation of a chylomicron flow blocking approach to investigate the intestinal lymphatic transport of lipophilic drugs. Eur J Pharm Sci. 2005;24(4):381-388. doi:10.1016/j. ejps.2004.12.006

28. Griffin BT, O’Driscoll CM. A comparison of intestinal lymphatic transport and systemic bioavailability of saquinavir from three lipid-based formulations in the anaesthetised rat model. J Pharm Pharmacol. 2006;58(7):917-925. doi:10.1211/jpp.58.7.0006

29. Chen JZ, Chou GX, Wang CH, et al. Characterization of new metabolites from in vivo biotransformation of norisoboldine by liquid chromatography/mass spectrometry and NMR spectroscopy. $J$ Pharm Biomed Anal. 2010;52(5):687-693. doi:10.1016/j.jpba.20 10.02.008
International Journal of Nanomedicine

\section{Publish your work in this journal}

The International Journal of Nanomedicine is an international, peerreviewed journal focusing on the application of nanotechnology in diagnostics, therapeutics, and drug delivery systems throughout the biomedical field. This journal is indexed on PubMed Central, MedLine, CAS, SciSearch ${ }^{\mathbb{}}$, Current Contents ${ }^{\mathbb{R}} /$ Clinical Medicine,

\section{Dovepress}

Journal Citation Reports/Science Edition, EMBase, Scopus and the Elsevier Bibliographic databases. The manuscript management system is completely online and includes a very quick and fair peer-review system, which is all easy to use. Visit http://www.dovepress.com/ testimonials.php to read real quotes from published authors. 